\title{
Retraction Note on: The Influence of Sodium Hyaluronate, l-Leucine and Sodium Taurocholate on the Nebulization of Aqueous Betamethasone-17-Valerate Suspensions
}

\author{
Mina I. Tadros ${ }^{1,2,3}$
}

Published online 17 June 2009

The article "The influence of sodium hyaluronate, 1-leucine and sodium taurocholate on the nebulization of aqueous betamethasone17-valerate suspensions" (Tadros MI. AAPS PharmSciTech. 2008;1:243-249) has been retracted at the request of the editor because it contains significant verbiage plagiarizing another publication: "Controlled drug delivery to the lung: Influence of hyaluronic acid solution conformation on its adsorption to hydrophobic drug particles" (IJP. 2007;330:175-182).

The online version of the original article can be found at http://www. springerlink.com/content/32584103843t1485/. This article should be considered as retracted.

\footnotetext{
${ }^{1}$ Department of Pharmaceutics and Industrial Pharmacy, Faculty of Pharmacy, Cairo University, Kasr El-Aini, Cairo, Egypt.

${ }^{2}$ The University of Iowa, Iowa City, IA, USA.

${ }^{3}$ To whom correspondence should be addressed. (e-mail: mina ebrahim@yahoo.com)
} 